\title{
An Efficient Binding Update Scheme Applied to the Server Mobile Node and Multicast in the MIPv6
}

\author{
Hye-Young Kim ${ }^{1}$ and Chong-Sun Hwang ${ }^{1}$ \\ Department of Computer Science and Engineering, \\ Korea University, Seoul, Korea \\ \{khy, hwang\}@disys.korea.ac.kr
}

\begin{abstract}
Mobile nodes are changing their point of attachment dynamically such as a network deployed in an aircraft, a boat, a train, or a car. These mobile nodes move together and share the same mobility properties. Therefore, this paper addresses an efficient location management scheme that utilizing for mobile nodes to move as a group. Our proposed method adds Server Mobile Node(SMN) to the existing Mobile IPv6 protocol, which maintains the functioning of mobile communication and stores the necessary information there. By implementing this method, it is expected that the lifetime of the mobile nodes re-setting efficiently. By combining the multicast routing with Mobile IPv6 method, in addition, the number of the binding upgrade(BU) messages produced in the home agent and CNs will not increase almost, although the number of the mobile nodes increases. We address the key functions for our proposed scheme including system configuration, location registration, and packet delivery. Our analytical model shows the usefulness of our proposed mechanism using analytical models and compares the results with the previous researches.
\end{abstract}

\section{Introduction}

In recent years, we have a rapid growth in the need to support mobile nodes(MNs) over global Internet-based mobile computing system. In addition, third-generation systems such as International Mobile Telecommunication System 2000(IMT-2000) and the Universal Mobile Telecommunications System(UMTS) seeks to unify existing cellular, Mobile IP cordless, and paging networks for universal use, the next generation will have the additional goal of offering heterogeneous services to mobile hosts that may roam across various geographical and network boundaries. In the system, MNs require special support to maintain connectivity as they change their point of attachment. When MN moves to another subnet out of its home network for smooth communication on the International Engineering Task Force(IETF)[1] Mobile Internet Protocol version $6(\mathrm{MIPv} 6)$ protocol, MN performs location registration to the home agent $(\mathrm{HA})$ and the correspondent nodes $(\mathrm{CNs})$ of $\mathrm{MN}$ even during its mobility 
by using the binding update(BU) messages in order to inform them of its current location after getting Care-Of-Address(COA)[2].

To support user's mobility, MN frequently creates the BU messages which in turn causes network overload because the additional signaling unnecessarily consumes the frequency bandwidth. The increase in the BU messages, therefore, has emerged as one of serious barriers to efficient location management. Therefore to deploy this mobile IP service widely, the Hierarchical Mobile Internet Protocol Version6(HMIPv6)[3] is also being researched in IETF. By adding a Mobility Anchor Point(MAP) in a visited network to manage local mobility there, we can limit HAs to providing only global or inter-MAP mobility management. HMIPv6 lets us to avoid frequent locational registration of MNs with HAs, which may be a long way from the MNs, and to reduce the time required for handovers. In the above approach to location management, the MNs are considered to move independently and so register their location with the network individually.

The evolution of information technology has enabled a mobile object such as person, car, bus, train, airplane, or ship to carry a plethora of information devices. Consider the situation in which a large number of MNs are riding on the same train or bus or aircraft. As it may contain a lot of mobile nodes, each communicating with several peers, the questions of locating, optimal routing and signaling overload are significantly more important. Therefore we consider an efficient location management scheme that mobile nodes move as a group. However, some CNs might even receive duplicate BU message carrying the same address in case they are corresponding with several MNs residing in the subnet. We therefore consider a solution based on multicast routing protocols for delivering network scope binding updates. In this paper, we propose a method to reduce the inefficient flood of BU messages by adding the Server Mobile Node(SMN) to the IETF MIPv6 protocol and by applying multicast to the transmission of the BU messages. As it provides a mobile networking function, an information keeping function, and a buffering function for the transmission packets, SMN performs the efficient setting of lifetime on the binding update messages and minimizes the delay or loss of transmitted packets. This can be applied to the transmission of the BU messages so that despite the increase of $\mathrm{MN}$ or $\mathrm{CNs}$, the number of the BU messages will not increase almost. Our proposed method minimizes the signaling due to the minimized addition of the BU messages and due to the expansion of MN.

The organization of this paper is as follows. This introduction section is followed by the literature review of related work. The section 3 is the main body of the paper, where we explain a new model for efficient location management, including a location registration scheme and its algorithm. The section 4 shows the mathematical modeling, its simulation, and the comparison of performance between the existing IETF Mobile IPv6 and the proposed new model. The final section suggests a future study direction. 


\section{Related Work}

IETF which is in charge of global standardization on the internet protocol has the Mobile IP Working Group to support connection and mobility through the internet of MNs. The recently provided MIPv6 protocol is registered as an internet draft and sometimes it is also adopted as the standard of IETF. However, signaling and required bandwidth created for mobility management on the MIPv6 protocol causes network overload, leading to traffic bottleneck. Particularly, it has been a concern that a registration process may cause too much traffic between the visited networks and home networks.

In order to resolve this problem, [3] and [4] have proposed a hierarchical mobility scheme based on MIPv6. Because of MIPv6's flexibility, their proposal can be deployed easily, and can interact and work well with MIPv6. In this scheme, a site can be an Internet Service Provider(ISP) network. A mobility network of a site is a LAN(Local Area Network) that defines an address space for the MNs roaming within a site. As routers for the MN, Mobility Servers(MSs) maintain a binding for every mobile node that currently visited the site. Hierarchical Mobile Internet Protocol version 6(HMIPv6), location registration is performed by sending the BU messages in accordance with changes in MN location, from the MS where they are currently encompassed to the top-ranked parent nodes of those MS, after MSs are tree-structured. In [5], by contract, the border router is placed for a communication with a separate mobility agent, and an external network for location management, based on MIPv6. In this case, location registration is performed by sending the BU messages only to the mobility agent in line with changes in MN location. In some studies [3, 4, 5], signaling is curtailed by reducing the number of the BU messages through the separation of the micro areas from the macro areas, considering the mobility of users on the MIPv6 and MIPv4 protocol. Each MN considers its location using location information from nearest MS or MAP, and then binding updates its location information individually if it detects that it has entered a new location registration area. Consider the very situation in which a large number of MNs within the MS or MAP are moving on the same mobility. All MNs try to access the network individually and simultaneously to update their location information. Therefore, they have inefficient flood of binding updates.

Concatenated Location Management(CLM) is used in [6] so that signaling created by the updated binding messages is minimized. Its basic idea is to treat MNs that share the same movement characteristics, as a single entity for location management. In order to update the location information of MN, it is to establish Intermediate Radio Stations(IRS) on each vehicle and CLM update the location information of all MNs with one action. So CLM, greatly enhanced the usage efficiency of the network since each group of users that moves together is treated as a single entity for location update. But [6] is considering only movement of vehicle or train which is a group of MNs without the respect to cases about individual MN's mobility.

Therefore, this paper proposes a method to efficiently create the BU messages by managing the local mobility of MNs similar to stratified MIPv6 by considering cases of mobility of MNs by adding SMN. 


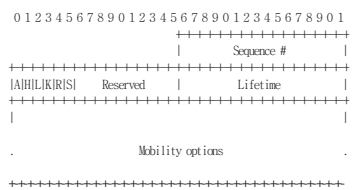

Fig. 1. BU Message Formats for Proposed System Configuration

Table 1. The Elements Consisting of SMN

\begin{tabular}{|l|l|l|l|l|l|l|l|l|l|l|}
\hline$S M N_{I P}$ & $C O A_{S M N}$ & $S M N_{P R E}$ & $T_{\mathrm{K} S}$ & $M N_{1}$ & $M N_{2}$ & $\ldots$ & $M N_{n}$ & $C N_{1}$ & $\ldots$ & $C N_{n}$ \\
\hline$\ldots$ & $\ldots$ & $\ldots$ & $\ldots$ & $\ldots$ & $\ldots$ & $\ldots$ & $\ldots$ & $\ldots$ & $\ldots$ & $\ldots$ \\
\hline$\ldots$ & $\ldots$ & $\ldots$ & $\ldots$ & $\ldots$ & $\ldots$ & $\ldots$ & $\ldots$ & $\ldots$ & $\ldots$ & $\ldots$ \\
\hline
\end{tabular}

\section{System Configuration}

\subsection{Proposed System Configuration}

Our proposed scheme manages network mobility and maintains binding information on MN by adding SMN to the IETF MIPv6 structure. One SMN has an area for location registration by the subnet, connected with many MNs. SMN is connected to wireless networks due to the mobility, for example, routers used on the aircraft, bus or train, it has a Server Gateway(SG) that manages network connection of SMN. HA that is in the home link of SMN will have the home address of SMN acquired upon location registration, and will have $C O A_{s m n}$ acquired when it moves to another subnet. The $S M N_{\text {prefix }}$ is a bit string that consists of some number of initial bits of the home address $S M N_{I P}$ which identifies the home link within the internet topology. All MNs share the same IP prefix[7]. The SMN performs location management through the BU messages received like MN does. As shown in Table 1, SMN keeps information about each of MNs for location management of MNs.

We add a new flag $(\mathrm{S})$ which is included in the BU message to indicate to the $\mathrm{HA}$ if the BU message is coming from a SMN and not from a MN. SMN provides connectivity to nodes in the mobile network, it indicates this to the HA by setting a flag $(\mathrm{S})$ in BU message as same scheme of Network Mobility(NEMO)[8]. The Figure 1 shows the BU message format in this paper. It sends the BU messages to HA and CNs of MN through SMN's SG right before the expiration of lifetime set in the BU messages and when it moves to another subnet. It is performed by the SMN, the COA would then be sent periodically to each $\mathrm{CN}$ corresponding with MNs. Therefore, the BU messages of SMN sent to CNs are exploded. Each $\mathrm{CN}$ would receive exactly the same $C O A_{s m n}$. However some $\mathrm{CNs}$ might even receive duplicate $\mathrm{BU}$ carrying the same $C O A_{s m n}$ in case they are corresponding with several MNs residing in the mobile network.

Therefore in our proposed system configuration, if IPv6 multicast is supported on network, SMN uses the method of sending the binding update mes- 


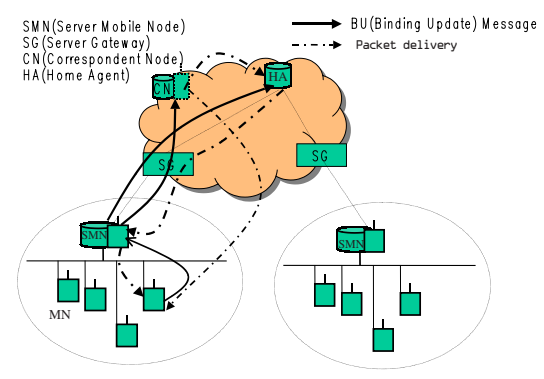

Fig. 2. Proposed System Configuration

sages to a Multicast Router(MR) that has a multicast group and multicast information to solve this problem. The subnetwork has a permanent multicast address which the SMN registers in the DNS[9, 10]. The SMN sends periodic BU s containing a binding between its $S M N_{\text {prefix }}$ and its $S M N_{C O A}$ to the multicast address. All CNs of MN use the IPv6 multicast method to join the multicast group. Many studies on the multicast method for MNs have been conducted $[11,12]$, but this subject is beyond the scope of the current study. Figure 2 shows the system configuration proposed in this paper.

\subsection{Location Registration}

SMN advertise an address on its subnet, and binds its current location with its own regional COA and list of the address prefixes of the links for the MNs. When MN moves to a new and different subnet, it gets two COAs. One is the SMN's $\operatorname{COA}\left(C O A_{S M N}\right)$ and the other is the MN's $\operatorname{COA}\left(C O A_{M N}\right)$ with a prefix equal to $C O A_{S M N}$. The MN registers the binding between its HA and the COAs, $\operatorname{COA}\left(C O A_{S M N}\right)$ and $C O A_{M N}$, to its HA and CNs through SMN. When the HA receives this BU message it create a binding cache entry binding the SMN's Home Address to it COA at the current point of attachment. The HA acknowledged the BU by a Binding Acknowledgement(BA) to the SMN through SG. A positive acknowledgement means that HA has set up forwarding for the network. Once the binding process completes, a bi-directional tunnel is established between the HA and SMN. The tunnel end points are SMN's Address and the HA's address. Then, when the HA receives packets for the MN, it can directly send them to the SMN by using an IP-in-IP tunnel. At that time SMN broadcasts the BU to MNs connected with it.

If MN moves within a domain, it only needs to change its $\left(C O A_{M N}\right)$, and the $\left(C O A_{S M N}\right)$ remains the same. Notice that it does not register any binding to its HA. MN has its own lifetime $T_{m}$ and lifetime $T_{s}$ of SMN. It is should be noted, however, that the determining of lifetime values and the frequency of binding refresh will also affect the signaling load of a network. If lifetimes have small values and the frequency of binding refresh is high, the signaling bandwidth for updating the COAs of an MN is also high. On the other hand, if the lifetimes have 
large values and the frequency of binding refresh is low, the signaling bandwidth for updating the COAs is also low. This reduction effect will increase as the number of MN increases. However, if long lifetime is set, MN stays in shorter time than lifetime set in a subnet. If MN moves to another subnet, this may cause the loss of packets because binding information kept by MN and CNs and that of HA sometimes have no information about MN location. MN takes steps to identify and authenticate the communication path and the communicating agent through lifetime set in its location registration[2]. If only lifetime of $\mathrm{MN}$ is simply set too long, therefore, increased are the chances of having a security problem between MNs and CNs. But in our proposed system configuration, MN's lifetime, $T_{m}$, becomes possible to set long in spite of no movement of $\mathrm{MN}$, because $\mathrm{BU}$ procedure is happened by lifetime of SMN. These lifetimes are initialized from the lifetime field continued in the BU and are decreased until it reaches zero. At this time, the entry must be deleted from the binding cache of CNs and the BU list of MNs. Because the MN requires the binding service from this node to go beyond this period, it must send a new BU to the HA, the SMN, and CNs before the expiration of the period, in order to extend the lifetime. Lifetime of SMN has to be the same as or longer than lifetime of MN. (That is, $T_{m} \leq T_{s}$ ).

MN's mobility in this proposed scheme can be classified into three cases and the proposed scheme for its location management is as follows:

Case 1. When MN moves within SMN

1) MN sends $B U_{a}$, BU messages, to SMN. $B U_{a}$ contains MN's home address, COA, COA with a prefix equal to $S M N_{\text {prefix }}$ and lifetime in the form of $\left\{M N_{I P}, C O A_{M N}, C O A_{S M N}, T_{m}\right\}$

2) SMN updates or creates the information list of MN kept as contents of $B U_{a}$, $\left\{M N_{I P}, C O A_{M N}, C O A_{S M N}, T_{m}\right\}$ and sends BA responding to $\mathrm{MN}$.

3) SMN sends the $B U_{a}$ of the CNs through the SG. The CNs create or update the MN's information.

Case 2. When MN moves to another SMN

1) MN sends $B U_{a}$, BU messages, to new $\mathrm{SMN}$ in the area it moved to. The MN obtains $C O A_{S M N}$ on the foreign link and $B U_{a}$ contains of its home address, COA, COA with a prefix equal to $S M N_{\text {prefix }}$ and lifetime in the form of $\left\{M N_{I P}, C O A_{M N}, C O A_{S M N}, T_{m}\right\}$ as information on MN.

2) SMN creates MN's information by using the contents of $B U_{a}$, and sends $B U_{b}$, BU messages, to $\mathrm{HA}$ of $\mathrm{MN}$ through $\mathrm{SG}$. A $B U_{b}$ contains $\left\{M N_{I P}, S M N_{I P}\right.$, $\left.C O A_{S M N}, C O A_{M N}, T_{s}, T_{m}\right\}$ as information on SMN.

3) HA updates or creates information as content of $B U_{b},\left\{M N_{I P}, S M N_{I P}\right.$, $\left.C O A_{S M N}, C O A_{M N}, T_{s}, T_{m}\right\}$, and sends BA, response to $B U_{b}$, to the SMN through the SG.

4) SMN sends a BA, response to $B U_{b}$, to the MN.

5) SMN sends a $B U_{b}$ to the CNs of MN through the SG. The CNs create or update the MN's information as content of $B U_{b},\left\{M N_{I P}, S M N_{I P}, C O A_{S M N}\right.$, $\left.C O A_{M N}, T_{s}, T_{m}\right\}$. 

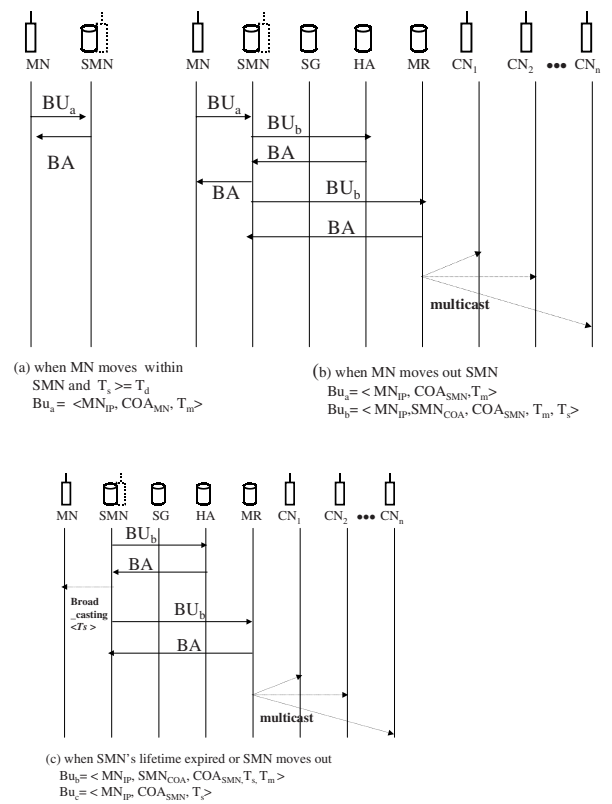

Fig. 3. BU Procedures of Proposed Scheme

Case 3. The expiration just before lifetime $T_{s}$ of SMN or when SMN moves

1) SMN sends $B U_{b}$, BU messages, to the HA of SMN through SG. As described in section 3.2, BU message is included a flag, $\mathrm{S}$.

2) HA updates or creates information as content of $B U_{b},\left\{M N_{I P}, S M N_{I P}\right.$, $\left.C O A_{S M N}, C O A_{M N}, T_{s}, T_{m}\right\}$, and sends BA, response to $B U_{b}$, to the SMN through the SG.

3) SMN broadcasts information as content of $B U_{c},\left\{S M N_{I P}, C O A_{S M N}, T_{s}\right\}$ to all connected MNs.

4) MNs of the SMN updates information kept as contents of $B U_{c}$.

5) SMN sends $B U_{b}$ to a CNs of SMN through the SG. The CNs create or update the MN's information as content of $B U_{b},\left\{M N_{I P}, S M N_{I P}, C O A_{S M N}\right.$, $\left.C O A_{M N}, T_{s}, T_{m}\right\}$.

Figure 3 is simple representation of the above explanation as a picture. Similar to [16], we define the following parameters for location update in the rest of this paper:

$C_{m s}$ : The transmission cost of location between the MN and the SMN

$C_{n g}$ :The transmission cost of location between the SMN and the SG.

$C_{s h}$ :The transmission cost of location between the SG and the HA.

$C_{h r}$ : The transmission cost of location between the HA and the MR.

$C_{r c}$ : The transmission cost of location between the MR and the CN.

$U_{s}$ : The processing cost of location update at the SMN.

$U_{g}$ : The processing cost of location update at the SG. 
$U_{h}$ : The processing cost of location update at the HA. $U_{r}$ : The processing cost of location update at the MR.

Here, If the MN moves in the SMN, it is referred to $C_{m n \_i n \_m o v e}$, and if the MN moves out of the current SMN to another SMN, it is referred to $C_{m m \_o u t}$ move, and if the SMN moves, it is referred to $C_{s m n}$. The following computations can apply to each case for one time BU cost. Since usually the costs for a wireless communication network is higher than a wire communication network, the ratio is considered by using $\rho$ to distinguish the difference between wire and wireless communication networks ( $\rho$ is dependent on the network, which is $\rho>1$ ) The communication cost for a wire communication network is referred to $\delta$, and the transmission cost for a wireless communication is referred to $\delta \rho$.

The multicast Listener Discovery(MLD) protocol[14] is used by IPv6 multicast routers to learn the existence of multicast group members on their connected links. The collected multicast group informantion is then provided to the multicast routing protocol. MLD is derived from version 2 of the Internet Group Management Protocol in IPv6. Therefore, we are considered by using $\gamma$ the cost of multicast in our proposed scheme. Because the SMN can be mobile, a communication network between the SMN and the SG is assumed to be a wireless one. Therefore, $C_{m s}, C_{s c}$, and $C_{s h}$ are the wireless communication networks, which incur most expensive communication costs. Because the number of hops or the distance between routers does not have much influence on the number of the BU messages or on costs, they are not considered in this paper. The following computations can apply to each case for one time BU cost.

$$
\begin{gathered}
C_{m n \_ \text {in_move }}=2 \delta \rho+U_{s} \\
C_{m n \_o u t \_m o v e}=8 \delta \rho+U_{s}+U_{g}+U_{h}+U_{r}+\gamma \cdot \delta \rho \\
C_{s m n}=7 \delta \rho+U_{g}+U_{h}+U_{r}+\gamma \cdot \delta \rho
\end{gathered}
$$

\subsection{Packet Delivery}

When the HA receives pakets for the MN, it can directly send them to the SMN by using an IP-in-IP tunnel. The SMN forward them to the MN by using another IP-in-IP tunnel, with the routing header option reffering to the binding entry in the SMN. If a packet with a source address belonging to the SMN's prefix is received from the network, the SMN reverse-tunnels the packet to the HA through this tunnel. This reverse-tunneling is done by using IP-in-IP encapsulation[15]. The HA decapsulates this packet and forwards it to the CN. For traffic or originated by itself, the SMN can use either reverse tunneling or route optimization as specified in [2].

When a data packet is first sent by a CN to a MN, it gets routed to the HA which currently has the binding for the SMN. When the HA receives a data packet meant for a MN, it tunnels the packet to SMN's current COA. The SMN decapsulates the packet and forwarding to MN. Then, the CN can send packets directly to the MN. 


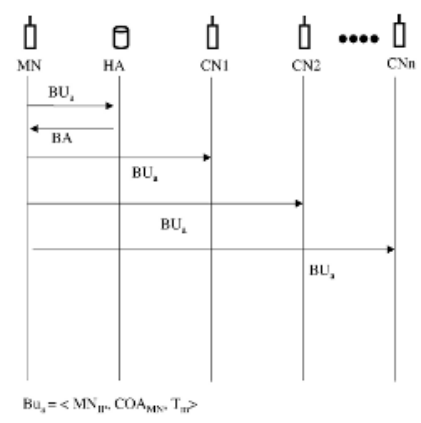

Fig. 4. BU Procedures of IETF Protocol

\section{Performance Analysis}

In this section, we make a performance analysis the proposed scheme in section 3. We compare the performance of Mobile IP and our proposed scheme.

\subsection{An Analytical Model of the Binding Update Costs in IETF MIPv6}

Costs for the binding update of mobile nodes incur when they move to another subnet and right before their lifetime expires. If the number of the CNs is referred to $c$, the binding update process by means of the MIPv6 protocol of IETF can be described as in Figure 4. As in the section 3.2, the following variables are used for computing costs for binding update.

$C_{m h}$ : The transmission cost of location between the MN and the HA.

$C_{m c}$ :The transmission cost of location between the MN and the CN.

$U_{h}$ : The processing cost of location update at the HA.

$U_{c}$ : The processing cost of location update at the CN.

Binding update costs for location registration when MN leaves its current location or right before expiration of its lifetime can be computed as follows:

$$
C_{m n \_i n \_m o v e}=2 \delta \rho+2 \cdot c \cdot \delta \rho+U_{h}+c \cdot U_{c}
$$

In this paper, we assume that MN that has lifetime $T_{m}$ moves on the path $R$ to compute total cost $C_{\text {tot_mip }}$ for binding update of MN on the MIPv6 protocol of IETF. The duration of time that MN stays in a location registration area is referred to a random variable, $T$.

Therefore, if an average of total costs for location update by the binding update messages that incur while MN stays in a location registration area is referred to $C_{\text {tot_mip }}$, it is shown as follows:

$$
C_{\text {tot_mip }}=\left(\left\lceil\frac{\frac{1}{\lambda}}{T_{m}}\right\rceil+1\right) \cdot 2 \delta \rho+2 \cdot c \cdot \delta \rho+U_{h}+c \cdot U_{c}
$$




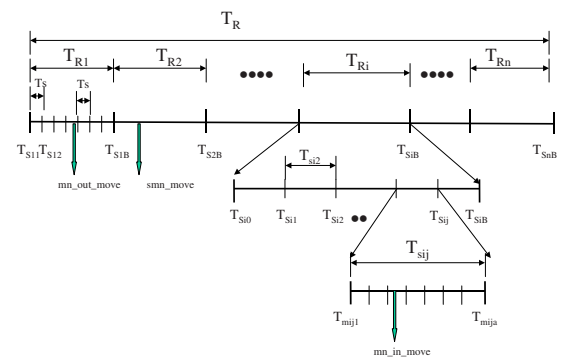

Fig. 5. The Relationship Between $T_{m}, T_{s}$ and $T_{R}$

\subsection{An Analytical Model of the Binding Update Costs in the Proposed Scheme}

To analyze the binding update costs of the proposed method in this paper, it is assumed that MN moves on the path $R$ that is divided into as many as $n$ of location registration areas. If the staying time of MN on the $i^{\text {th }}$ path, $R_{i}$ is shown as, $T_{R}$, staying time of MN, $T_{R_{i}}$, follows the exponential distribution that has the average $\frac{1}{\lambda} \cdot T_{R_{i}}$ is assumed. $T_{s}$ represents the binding lifetime of SMN in a location registration area. $T_{m}$ represents MN's binding lifetime. The relation with $T_{R_{i}}$, $T_{s}, T_{m}$ is $T_{R_{i}} \geq T_{s} \geq T_{m}(1 \leq i \leq n)$, and in the case of $T_{R_{i}}<T_{s}<T_{m}$, it is excluded from the analytical model because no binding update occurs. $T_{s}=\alpha \cdot T_{m}$ (provided that $\alpha \geq 1$ ), $T_{R_{i}}=\beta_{i} \cdot T_{s}$ (provided that $\beta_{i} \geq 1$ ) is assumed. $T_{s_{i j}}$ represents the $j$ th $T_{s}$ section of SMN in the path $R_{i}$. (provided that $j \geq 0$ ) That leads to $\sum_{i=0}^{n-1} T_{R_{i}}+$ $T_{s} \leq T_{s_{i j}}<\sum_{i=0}^{n-1} T_{R_{i}}+j \cdot T_{s}$ represents the $k^{t h} T_{m}$ section within $T_{s_{i j}}$, SMN's binding lifetime. That leads to $(k-1) \cdot T_{m} \leq T_{m_{i j k}}<k \cdot T_{m}$ within $T_{S_{i j}}$. The formula for time required for $\mathrm{MN}$ to move on the path can be shown as Figure 5, it becomes, $T_{R}=\sum_{i=1}^{n} T_{R_{i}}, T_{R_{i}}=\sum_{j=1}^{\beta_{i}} T_{s_{i j}}, T_{s_{i j}}=\sum_{k=1}^{\alpha} T_{m_{i j k}}$ assume that the initial state refers to time when MN in possession of binding lifetime $T_{s}$ stays within a SMN on the path $R_{i}$ for cost analysis. If MN's staying time, $T_{R_{i}}$ on $R_{i}$ is shown as random variable $X$, as assumed above, if $X$ follows the exponential distribution that has the average $\frac{1}{\lambda}$, the Cumulative Density Function $F_{i}(t)$ for the probability that MN stays for the time less than within its moving area $R_{i}$ is shown as follows:

$$
F(x)=P(X \leq x)=\int_{x=0}^{x=T_{R i}} \lambda \cdot e^{-\lambda x} d x
$$

An analysis model of binding update cost for MN's movement within the given SMN. If the probability that MN within the $j^{\text {th }}$ binding lifetime section of SMN on the path $R_{i}$ stays in the $k^{t h}$ binding lifetime section $(k \geq 1)$ is $P(k-1, k)$. Before MN that stays in the $j^{t h}$ binding lifetime section of SMN exceeds the $k^{t h}$ binding lifetime section on the path $R_{i}$, the frequency of the BU messages caused by MN becomes $k-1$. The average frequency of location 
update that is caused by MN within the $j^{\text {th }}$ binding lifetime section of SMN can be expressed as follows:

$$
\begin{aligned}
& P(0,1)=P_{r}\left[0 \leq X<T_{m}\right]=1-e^{-\lambda \cdot T_{m}} \\
& \sum_{k=1}^{\alpha-1} k \cdot P(k-1, k)=\left(e^{-\lambda \cdot T_{m}}+\left(e^{-\lambda \cdot T_{m}}\right)^{2}+\cdots+\left(e^{-\lambda \cdot T_{m}}\right)^{\alpha-1}\right) \cdot P(0,1) \\
& \quad=e^{-\lambda \cdot T_{m}} \cdot\left(1-\left(e^{-\lambda \cdot T_{m}}\right)^{\alpha-2}\right)
\end{aligned}
$$

The BU costs of the MN's movement only within SMN can be shown as in Formula(1) of 3.2. Therefore, $E_{\left[m n_{-} i n_{-} m o v e\right]}$, the average total costs for MN's location update within only SMN are as follows:

$$
\begin{aligned}
E_{\left[m n \_i n \_m o v e\right]} & =\sum_{k=1}^{\alpha-1} k \cdot P(k-1, k) \cdot B U \operatorname{cost}=e^{-\lambda \cdot T_{m}} \cdot\left(1-\left(e^{-\lambda \cdot T_{m}}\right)^{\alpha-2}\right) \\
& \cdot C_{m n \_i n \_m o v e}
\end{aligned}
$$

An analytical model of the binding update costs for moving to another SMN after staying within the given SMN. MN is assumed to be within the $j^{\text {th }}$ binding lifetime of SMN on the path $R_{i}$. Before MN within the $j^{t h}$ binding lifetime section of SMN on the path $R_{i}$ moves to another SMN that exceeds the $k^{t h}$ binding lifetime, the frequency of BU messages caused due to its moving to another SMN becomes $k-1$. Therefore, the average frequency of location update caused by the movement of MN to another SMN within the $k^{t h}$ binding lifetime section of the SMN can be expressed as follows:

$$
\begin{gathered}
\sum_{k=1}^{\alpha-1} k \cdot Q(j, k-1, k)=\sum_{k=1}^{\alpha-1} k \cdot e^{-\lambda \cdot(k-1) \cdot T_{m}} \cdot Q(j, k-1, k) \\
=e^{-\lambda \cdot T_{s}} \cdot\left(1-\left(e^{-\lambda \cdot T_{m}}\right)^{\alpha-2}\right)
\end{gathered}
$$

When MN in possession of binding lifetime $T_{m}$ within the $j^{t} h$ binding lifetime section of SMN on the path $R_{i}$ moves to another SMN on the path $R_{i}$, if the frequency of its movement is $w$, the frequency for $T_{m}$ results in $r=\frac{w}{\frac{T_{R_{i}}}{T_{m}}}$ and because the assumption based on 4.2 leads to $T_{R_{i}}=\alpha \cdot \operatorname{beta}_{i} \cdot T_{m}, r \stackrel{\frac{m}{=}}{=} \frac{w}{\alpha \cdot \beta_{i}}$, results. The binding update costs for MN caused by the movement of MN to another SMN within the $k^{\text {th }}$ binding lifetime section of SMN can be shown as in formula(2) of the section 3.2. The average total costs of location update $E_{\left[m n_{\text {_out_move }}\right]}$ that are incurred because MN within the $j^{\text {th }}$ lifetime section of SMN moves to another SMN are as follows:

$$
\begin{aligned}
E_{\left[m n_{-} \text {out_move }\right]} & =\sum_{k=1}^{\alpha-1} Q(j, k-1, k) \cdot k \cdot \text { frequency } \cdot B U \text { cost } \\
& =e^{-\lambda \cdot T_{s}} \cdot\left(1-\left(e^{-\lambda \cdot T_{m}}\right)^{\alpha-2}\right) \cdot \frac{w}{\alpha \cdot \beta_{i}} C_{m n \_o u t \_m o v e}
\end{aligned}
$$


An analytical model of the binding update costs for moving to another SMN after staying within the given SMN. When SMN moves from $j-1$ to $j$ of binding lifetime $T_{s}$ on the path $R_{i}$, the frequency of location update by the binding update messages of MN created in the movement becomes $j-1$. Therefore, the number of location update by the MN's binding update messages are as follows:

$$
\sum_{j=0}^{\beta_{i}-1} j \cdot R(j-1, i)=e^{-\lambda \cdot T_{s}} \cdot\left(1-\left(e^{-\lambda \cdot T_{s}}\right)^{\beta_{i}-2}\right)
$$

When SMN moves from $j-1$ to $j$ of binding lifetime $T_{s}$ on the path $R_{i}$, the MN's binding costs incurred in this movement can be shown as in Formula(3) of the section 3.2. Therefore, the average total costs for location update by the MN's binding update messages created, $E_{\left[m n_{-} \text {out_move }\right]}$ are as follows:

$$
\begin{aligned}
E_{\left[s m n \_m o v e\right]} & =\sum_{k=1}^{\beta_{i}-1} j \cdot R(j-1, j) \cdot B U \text { cost } \\
= & e^{-\lambda \cdot T_{s}} \cdot\left(1-\left(e^{-\lambda \cdot T_{s}}\right)^{\beta_{i}-2}\right) \cdot C_{\text {smn_move }}
\end{aligned}
$$

Accordingly, if the total average costs for location registration by the MN's binding update messages in the proposed method are referred to $E_{[T O T]}$

$$
\begin{aligned}
& E_{[T O T]}=e^{-\lambda \cdot T_{m}} \cdot\left(1-\left(e^{-\lambda \cdot T_{m}}\right)^{\alpha-2}\right) \cdot C_{m n_{-} \text {in_move }} \\
& \quad+\quad e^{-\lambda \cdot T_{s}} \cdot\left(1-\left(e^{-\lambda \cdot T_{m}}\right)^{\alpha-2}\right) \cdot \frac{\omega}{\alpha \cdot \beta_{i}} C_{m n \_o u t \_m o v e} \\
& \quad+\quad e^{-\lambda \cdot T_{s}} \cdot\left(1-\left(e^{-\lambda \cdot T_{s}}\right)^{\beta_{i}-2}\right) \cdot C_{\text {smn_move }}
\end{aligned}
$$

\subsection{Comparison and Analysis for Binding Update Costs}

We have analyzed the binding update costs of MN depending on the number of $\mathrm{CNs}$, the number of MNs, the mobility of MN, and the progression of time, and compared them with IETF MIPv6. The the results were computed by MATLAB, and Table 2 lists some of the parameters used in our performance analysis[16].

Table 2. The Elements consisting of SMN

\begin{tabular}{|l|l|l|l|l|l|l|l|l|l|l|l|}
\hline$U_{s}$ & $U_{h}$ & $U_{c}$ & $U_{r}$ & $U_{g}$ & $\mathrm{~N}$ & $\rho$ & $\gamma$ & $\alpha$ & $\beta$ & $\mathrm{M}$ & $\mathrm{C}$ \\
\hline 15 & 25 & 10 & 10 & 10 & 10 & 10 & 260 & 2 & $1 . .10$ & 15 & 5 \\
\hline
\end{tabular}

In the Table 2, $m$ represents the number of MNs, $n$ represents the maximum number of paths on which MN moves, and $c$ represents the maximum number of CNs, $\alpha$ and $\beta$ represent the update frequency of MN and the sever moving nodes while MN stays within a SMN on a given path. Besides, $\rho$ is a variable that distinguishes the difference in communication costs between wire and wireless communication networks. 


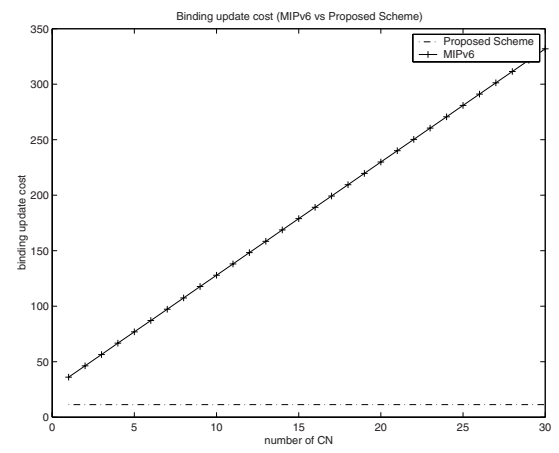

(a) Increase of Number of CN

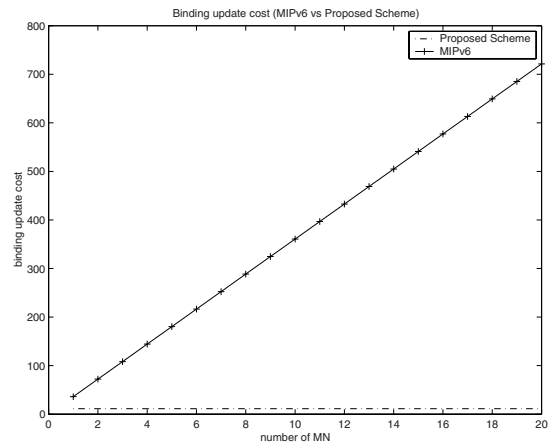

(b) Increase of Number of $\mathrm{MN}$

Fig. 6. Binding Update Cost

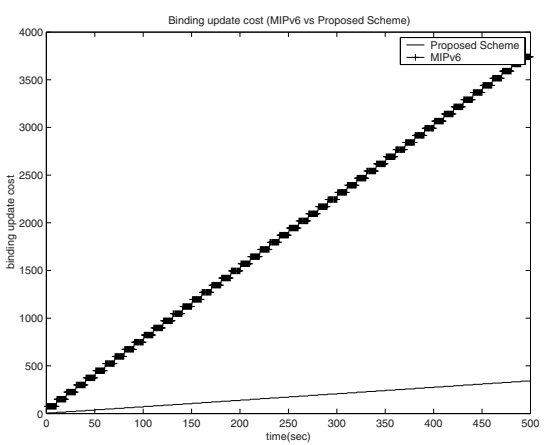

(a) $\lambda=0.01$

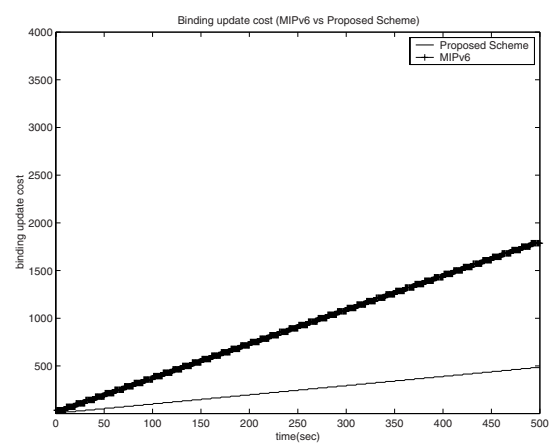

(b) $\lambda=0.06$

Fig. 7. Comparision of Binding Update costs According to Mobility

$\gamma$ is a variable for multicast costs in network. On each link, one multicast router is elected to act as a querier. It periodically sends out a query onto this link. The default value for the query interval is 125 second. And the default value of the maximum response delay is 10 second. A group membership timer is using the default values for, 2 - query + response delay, results in a default value 260 second[17].

$U_{s}, U_{h}, U_{r}, U_{g}$ and $U_{r}$ mean costs for location update performance from SMN, HA, and the multicast router, respectively. First, MN's binding update costs have been computed by increasing the number of CNs from 1 to 30 .

If the multicast router is supported as in Figure 6 (a), the BU coast in our proposed scheme is not increase almost by the increased number of CNs. It shows a growing difference in the BU costs as the number of CNs increases, compared 
with the protocol of IETF MIPv6. The MN's binding update costs are also computed, as the number of MN connected to HA on the IETF MIPv6 protocol and the number of MN connected to SMN are increased up to 20. The BU coast in our proposed scheme is not increase almost by the increased number of $\mathrm{MN}$ as in Figure 6 (b). To analyze the MN's BU costs according to MN's mobility, $\lambda$ of $\frac{1}{\lambda}$ the average staying time of MN is changed for the MN's BU costs in the cases of 0.01 and 0.06 . As is smaller, MN's mobility becomes frequent. When compared with IETF MIPv6 as in Figure 7 (a) and (b), it is known from frequent MN's movement and the section of $\lambda=0.01$ that our proposed method is more efficient. Besides, when time for MN is increased, it is shown that the BU costs are not increase almost.

\section{Conclusion and Future Work}

This paper adds SMN that has mobility on the IETF MIPv6 protocol, and considers two lifetimes.(i.e., MN's lifetime and SMN's lifetime)

Presented in this paper is a technique that can minimize costs for MN's binding update by applying the multicast method with the multicast router for CNs. Also, we propose the location update methods about each case which BU of MN happens, we calculate location update costs to be required in each cases. Presented in this paper is a technique that can minimize costs for MN's BU by applying the multicast method with the multicast router for $\mathrm{CN}$ and adding the SMN. And we calculate location update costs each technique of IETF MIPv6 and our proposed method and do the comparison analysis. Our proposed method is maintained without taking the influence almost by the increased number of MNs. If the staying time of MN follows the exponential distribution that has the average $\frac{1}{\lambda}$, as $\lambda$ is smaller, MN's mobility becomes frequent. When compared with IETF MIPv6, it is know from frequent MN's movement that our proposed method is more efficient.

Consequently our proposed method, when the number of MN increases, and when MN has frequent mobility, is efficient.

\section{References}

1. The Internet Engineering Task Force

2. D. B. Johnson and C. Perkins, "Mobility Support in Pv6", IETF(International Engineering Task Force) Internet Draft, draft-ietf-mobileip-ipv6-24.txt, June 30, 2003

3. H.soliman, C.Castelluccia, K. El-malki, and L. Beller, "Hierarchical MIPv6 mobility management(HMIPv6)", dfraft-ietf-mobileip-hmipv6-06.txt, July 2002

4. C.Castelluccia, "HMIPv6: A hierachical mobile ipv6 proposal", ACM Mobile Computing and Communication Review, vol. 4, no. 1, pp.48-59, Jan. 2000

5. C. E. Perkins, "Mobile-IP Local Registration with Hierarchical Foreign Agents", Internet Draft-perkins-mobileip-hierfa-00.txt, Feb. 1996. 
6. Hideaki YUMIBA, Koji SASDA, and MAsami YABUSAKI, "Concatenated Location MAnagement", IEICE Transaction Communication Vol. E85-B, No. 10, pp. 2083-2089, 2002

7. Thierry Ernst, Alexis Olivereau, Ludovic Bellier, Claude Castelluccia, Hong-Yon Latch, "Mobile Networks Support in Mobile IPv6(Prefix Scope Binding Updates)", Internet-Draft draft-ernst-mobileip-v6-network-03.txt, Internet Engineering Task Force(IETF), March 2002

8. Vijay Devarapalli, Ryuji Wakikawa, Alexandru Petrescu and Pascal Thubert, "Network Mobility(Nemo) BAsic Support Protocol", IETF Internet draft, dfraft-ietfnemo-basic-support-02.txt, Dec. 2003

9. Levon Esibov, Bernard Aboda, Dave Thaler, "Linklocal Multicast Name Resolution(LLMNR)", Internet-Draft draft-ietf-dnsext-mdns-30.txt, DNSEXT Working Group Internet Engineering Task Force(IETF), 17 March 2004

10. B.Haberman, J.Martin,"Multicast Router Discovery", MAGMA Working Group IETF Internet draft, dfraft-ietf-magma-mrdisc-00.tat, Feb. 2004

11. R. Vida, L.Costa, "Multicast Listener Discovery(MLDv2) for IPv6", Internet-Draft draft-vida-mld-v2-08.txt, Internet Engineering Task Force(IETF), June 2004

12. George Xylomenos, George C.Polyzos, "IP Multicast for Mpbile Hosts", IEEE Communication Magazine, Vol 35, Issue 1, pp. 54-58, Jan. 1997

13. RalphDroms, C. Perkins, Jim Bound, M. Carney, "Dynamaic Host Configuration Protocol for IPv6", INTERNET-DRAFT,jdraft-ietf-dhc-dhcpv6-20.txti, September 2001.

14. S.Deering, W.Fenner, and B.Haberman, "Multicast Listener Discovery(MLD) for IPv6", RFC 2710. Oct. 1999

15. A.Conta and S. Deering, "Generic Packet Tunneling in IPv6 Specification", RFC 2473, IETF December 1998

16. Jiang Xie, Lan F. Akyildiz, "A Novel Distributed Dynamic Location Management for Minimizing Signaling Coasts in Mobile IP", IEEE Transaction on Mobile Computing vol, 1, No. 3, pp. 163-175, 2002

17. Christian Bettstetter, Anton Riedl, Gerhard GeBler, "interoperation of Mobile IPv6 and Protocol Independent Multicast Dense Mode" 